\title{
Genetic Algorithm based Solution Model for Multi-Depot Vehicle Routing Problem with Time Windows
}

\author{
A. Ramalingam ${ }^{1}$, K. Vivekanandan ${ }^{2}$ \\ Associate Prof, Department of MCA, Sri ManakulaVinayagar Engineering College, Puducherry, India ${ }^{1}$ \\ Professor, Department of Computer Science and Engineering, Pondicherry Engineering College, Puducherry, India ${ }^{2}$
}

\begin{abstract}
We present a novel Ordered Distance Vector (ODV) based Equi-begin with Variable-diversity (EV) Technique for the exact solution of a variation of the vehicle routing problem with time windows in which the transportation fleet is made by vehicles with different capacities and fixed costs, based on different depots. The MultiDepot Vehicle Routing Problem with Time Windows (MDVRPTW) is a generalization of the standard Vehicle Routing Problem (VRP). The VRPTW is NP-Complete. The MDVRPTW problem is addressed using an efficient Genetic Algorithm (GA). Genetic algorithm is a powerful optimization technique to solve NP-Complete problems. In GA different initial population seeding techniques were used to find out the performance of an individual. In this paper, we are analyzing the performance of Gene Bank technique with a proposed novel Ordered Distance Vector (ODV) based EV Technique in terms of convergence rate (\%), quality solution and convergence diversity. Different authors provided different bench mark instances for MDVRPTW in neo research group. In order to compare the effectiveness and performance of the proposed population seeding technique, we are using Cordeau's benchmark instances; it contains 20 different instances of MDVRPTW obtained from VRPLIB were experimented using MATLAB software.
\end{abstract}

Keywords - Multi-Depot Vehicle Routing Problem with Time Windows, ODV-EV Technique, Convergence, Performance, Diversity, Genetic algorithm, VRPLIB, MATLAB

\section{INTRODUCTION}

Vehicle routing problem (VRP) is a very important Density of goods to be delivered etc. The beginning and combinatorial optimization problem. The subject is simple end points of the vehicle is called the depot. The shortest to explain however tough to attain an optimum resolution distance travelled by all the vehicle is that the feasible because of the high process quality [1]. Since the matter is solution [1].

expounded with single depot, the vehicle routing problem Each vehicle will carry a restricted weight and just one is additionally named Single-depot vehicle routing vehicle is allowed to go to every customer.VRP could be a problem. Single-depot vehicle routing problem aren't NP-hard problem that is troublesome to unravel and are appropriate for sensible things. Vehicle routing problem often ready to solve the problem in polynomial time [7,8]. with over one depot are called Multi-depot Vehicle Meta heuristics are ways won't to notice the top quality Routing problem [2]. The Multi-Depot Vehicle Routing solutions [5,6]. Genetic Algorithms (GA) are performing Problem (MDVRP), an extension of classical VRP, may to solve the Vehicle Routing problem. In Genetic be a NP-hard problem for at the same time determinative algorithmic program provides the resolution by the routes for many vehicles from multiple depots to a performing crossover and mutation operators that collection of customers and come back to a respective simulates the reproduction [7,8]. The fitness values are depot. The target of the problem is to seek out routes for calculated for selection a solution from the population [9]. vehicles to service all of customers at a nominal cost in The Fig 1 shows the instance of VRP with three routes and terms of variety of routes and total travel distance, and at single depot. Let's assume that we have 3 trucks to drop the same time not violating the capability and time period off to these nine service destinations and all starts from constraints of the vehicles [3,4].The VRPTW is the same "Depot" and the best route for these three trucks to problem with the additional restriction that in VRPTW a minimize the total distance driven.

time window is associated with each customer, The aim is to minimize the vehicle fleet and the sum of travel time and waiting time needed to supply all customers in their required hours.

\section{VEHICLE ROUTING PROBLEM}

The main aim of the VRP is that the set of vehicles must visit a group of customers/ cities specifically once and come to the place to begin with minimal price .The cost parameters may be Distance of travel, Time of travel, 


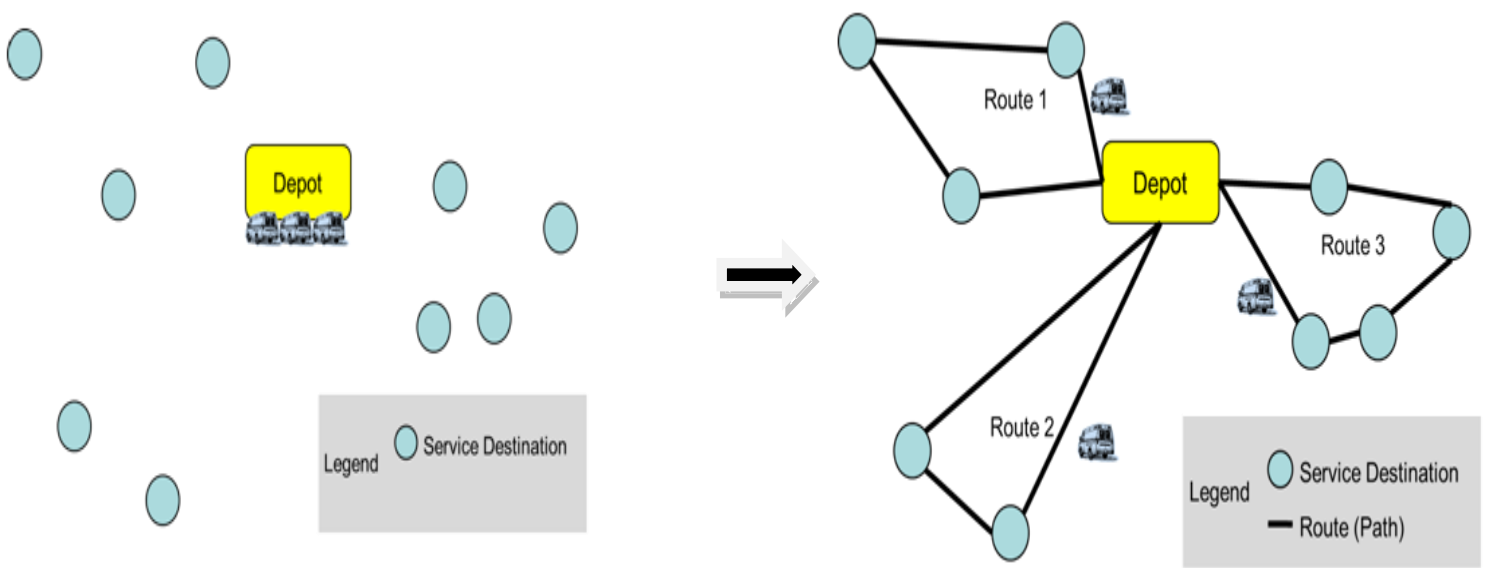

Fig. 1 An instance of VRP with Three Routes and one Depot

The aim is to reduce the vehicle fleet and also the total of time period and waiting time required to produce all customers in their needed hours [11].

\section{MULTI-DEPOT VEHICLE ROUTING PROBLEM}

Multiple Depot Vehicle Routing problem (MDVRP) is that the variant of vehicle Routing problem (VRP). MDVRP is same as VRP however with more than one depot [10]. An organization could have many depots from that it will serve its customers. If the customers are clustered around depots, then the distribution problem ought to be shapely as a collection of freelance VRPs [18]. However, if the customers and therefore the depots are blended then a Multi-Depot Vehicle Routing problem ought to be solved. A MDVRP needs the assignment of customers to depots. A fleet of vehicles is predicated at every depot. Every vehicle originates from one depot, service the customers allotted thereto depot, and come back to identical depot[17]. Multiple depots only stipulate that there are multiple sources. The instance of Multi Depot Vehicle Routing Problem with Time Windows (MDVRPTW) consists of four routes and a four depot with solution is as shown in the Fig. 2.

The Main aim is to attenuate the vehicle fleet and also the total of time period, and also the total demand of commodities should be served from many depots. This attenuates the vehicle fleet and the total of amount, and so the whole demand of commodities ought to be served from several depots. A solution is feasible if every route satisfies the quality VRP constraints and begins and ends at identical depot.

\section{MULTI-DEPOT VEHICLE ROUTING PROBLEM WITH TIME WINDOWS - MODEL AND EXPLANATION}

In this model, In MDVRPTW is considered a set of vehicles with indistinguishable capacity in order to deliver the goods to known customer demands from a multiple depots at minimum transportation cost. The Multi Depot
Vehicle Routing problem with Time Windows (MDVRPTW) is taken into account for a sensible description of transportation coming up with. The MDVRPTW describes the matter to deliver uniform goods to a collection of customers from a collection of depots with vehicles with heterogeneous capacities [15,17]. The delivery has to be done at intervals, customer-specified time window and also the vehicles ought to come back to identical depot wherever they need started. Every customer has got to be delivered once [12].

\section{MDVRPTW - SOLUTION FORMULATION}

The objective of MDVRPTW is to discover a possible set of tours with the minimum total traveled distance. The MDVRPTW optimal solution should satisfies the following conditions:

(a) Each route should have different depots and each route should starts and end at the same depot.

(b) The total capacity of the vehicle should not exceed the total demands of the customers in that route.

(c) Each route must depart and terminate within the time window associated with the depot.

(d) Exactly one vehicle has to visit each customer and service their demands

(e) The total cost of all tours has to be minimized.

VII.

\section{ALGORITHM DESIGN AND DEVELOPMENT}

A Genetic algorithm (GA) has been proposed to solve the MDVRPTW. However, completely different genetic operators applied to improve the efficiency of the MDVRPTW of the population in every generation. Moreover, the varied population seeding techniques are altered based mostly on the problem and applied with the improved GA.

\section{A. Selection}

Even although, the optimal solutions have been determined for every generation, the individuals within the population are altered because of the genetic operations as a result there's an opportunity of 

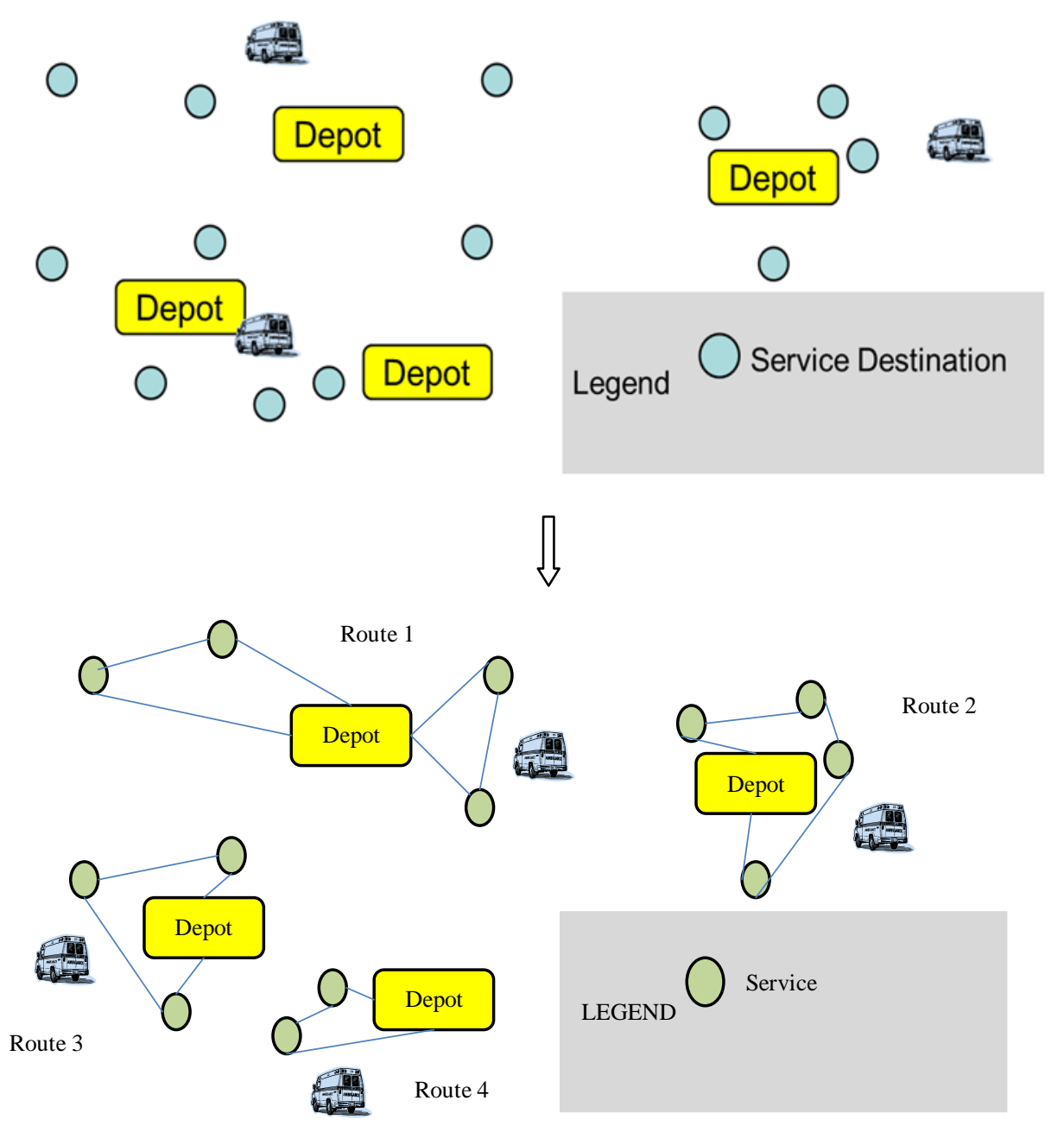

Fig. 2 A Solution Model for MDVRP with 4 depot and route

modifying the optimal solution in next generation. Consequently, the preservation of optimal solutions, has required, so the total cost of every individual in the population is determined w.r.t to the objective function $[1,13]$. The individuals those having least distance are selected as a moralist individual primarily based on the moralist rate $[7,8]$. The moralist individuals are passed to succeeding generation with none modification of further genetic operations.

\section{B. Crossover}

The crossover method is accomplished through greedy crossover [7,8]. In crossover, choose any two random Parent individuals from the whole population. The leading customer in the parent individual is moved and Assign to the offspring individual current customer[1,13]. currently estimate the position of the current customer in each the parent individuals and so find the proper facet and left facet customer to the current customer in each the parent individuals. Note that, if the position of current customer is starting customer then the position of left customer to the present customer is last customer within the individual and if the position of current customer is last client then the position of Righ customer to the current customer is last customer.

\section{Mutation}

Finally, it is valuable to have a Mutation process, Generate two random locations of the customer in the individual, that is having same demand and then swap the customers in the offspring individual in their various positions and vice versa.

Succeeded by mutation method offspring individual to have been displaced to the population set, execute this method until the population makes the most population size. Evaluate the entire value for all the individuals from the whole population, it is necessary to add the depot at the start and also the finish of the every route in the individuals whereas calculating the cost[4,5,13,16].A generalized equation for calculating the cost of the routes present in all the individuals. However, the objective of CVRP is that to reduce the cost of the total value of the routes gift in the individuals with a single constraint the total demand of the route shouldn't exceed the limit of the capability of the vehicle[1,3,4,6].Objective function of the problem is to find the optimal solution, which is given as,

Optimal Solution $\leftarrow \min \{T C\}$, where $T C$ the total cost has to be minimized. 


\section{EXPERIMENTAL TECHNIQUES USING ODV-EV POPULATION SEEDING TECHNIQUE}

An ODV-EV technique is a current population seeding technique, this methodology used to quantify the individuals in the population. Since all the routes starting from the different depot, ODV-EV technique is used for population seeding process.

In this section, a transient description on alternative population seeding techniques like Gene Bank Technique and that has been studied. The techniques are compared with Ordered Distance Vector (ODV) based Equi-begin with Variable-diversity (EV) technique.

\section{A. Gene Bank (GB) technique}

This technique is proposed to generate the initial population of solutions with quality and diversity. Before forming the initial population, the adjacent/ nearest gene of each gene based on the problem solution is kept in the gene bank. (i.e.) In the case of TSP, the next two nearest customer based on the distance is kept in the gene bank. An initial gene $\mathrm{x}$ in the individual is initialized randomly, then select the next nearest gene from the gene bank randomly for the gene $\mathrm{x}$. the nearest gene is added in the individual, if the nearest gene is already present in the individual then the next gene is chosen randomly from the set of unallocated genes. This method is repeated until we generate the problem solution.

\section{B. Algorithm for MDVRPTW using gene bank population seeding technique}

Gene bank technique is a population seeding technique, in this approach the genes are kept in the gene bank. While constructing the individuals in the population the genes are taken from the gene bank and subsequently the beginning for all the routes has have different depots. Ultimately the algorithm continues with different parameters as like in ODV-EV technique; n number of customer, Popsize total number of individuals in the population, $\delta$ capacity of vehicle and $\beta$ 'gene bank ratio'. The distance matrix $\mathrm{DM}_{\mathrm{n} \times \mathrm{n}}$ of each customer to other customers are estimated from the benchmark datasets and transmitted to a temporary distance matrix. At this instant gene bank started to construct, for each customer the ' $\beta$ ' number of adjacent customers is assessed and then considered to move to the $\mathrm{GB}_{\text {Cust } \times \mathrm{i}}$ matrix; this process has been repeated until the gene bank ratio $i<=\beta$. At each time the vehicle has to start from the depot, so that the route is initially assigned with random depot Init_C $\leftarrow$ $R A N D(M D)$. The iteration to construct the individuals in the population has been started and the total demand of the route has been initialized. Based on the gene bank ratio' $\beta$ ', A random number from 1 to $\beta$ has been generated. The customer in the location of ' $\beta$ ' has selected from the GB matrix with respect to the current customer. The selected customer is picked as next customer Next_C $\leftarrow G B_{\text {Cur_C,Rand }}$, afterwards check whether the next customer already present in the individual $\left\{\right.$ Indiv $_{\text {Size }}:$ Next_C $\in$ Indiv $\left._{\text {Size }}\right\}$ and the current time window should not goes beyond the ending time of the customere Cur_C $_{-} \leq t w \leq l_{\text {Cur_c }}$. Estimate the demand of the next customer Cur_Demand $\leftarrow$ Demand ${ }_{\text {Next_C }}$ then added to total demand $T_{-}$Demand $\leftarrow T_{-}$Demand + Cur_Demand.

Nevertheless in the MDVRPTW, the primary constrains is that the total demand of the route should not exceed the capacity of the vehicle $T_{-}$Demand $\leq \delta$, so that the total demand of the route is matched with the capacity of the vehicle. If the constrain is satisfied, the vehicle has to return to the same starting depot and a new route is allocated to the vehicle from a different random depot; if not then the customer has been added in the individual Indiv $v_{\text {len }} \leftarrow$ Next_Cand increase the length of the individual len $\leftarrow$ len +1 . Repeat this step until a complete individual is formed len $\leq n$, then the complete individual is moved to the population. Explained above process has been repeated until the entire population reaches the population limit Size $<=$ PopSize. Population generated by this method is transfer for further process and forwarded to the enhanced genetic algorithm.

C. Object distance vector based Equi-begin with Variablediversity $(E V)$ technique

ODV primarily based EV technique is an efficient technique to initialize the initial population, there are totally different type of ODV technique ,since beginning city is fixed, we tend to are moving to The EV (Equibegin with Variable-diversity) primarily based ODV (Ordered Distance Vector) population seeding technique supported the ODV matrix. Ordered Distance Vector population seeding technique consists of two stages. First, an Order Distance Matrix (ODM) are going to be created from the Distance Matrix (DM) and followed by generating the initial population supported the ODM $[1,13,8,14]$. Ordered Distance Vector primarily based population seeding technique generates a group of permutation of ' $n$ ' cities using the Ordered Distance Vector matrix. In every permutation, the sequence of the cities is chosen such the sum of distances between the cities is close to minimum. This methodology is efficient because of the simplest adjacent $(b a)$ value; it's used to choose consequent city in population generation. Consequent customer within the individual is additional on the bax value, bax is a number that choosing within the range of best adjacent value $b a$. The individuals within the population have high permutation of cities and therefore the time complexity may be reduced.In ODV the customers are organized based on the distance that is calculated by the permutation of Cust $_{1}$, Cust $_{2}, \ldots$ Cust $_{n}$ customers. The ODV of a Cust Is, $_{x}$, oDV $\left(\right.$ Cust $\left._{x}\right)=$ Cust $_{y}$, Cust $_{y+1}$, . Cust $_{n-1}$

Then, $\left(\right.$ Cust $\left._{x, y}\right) \leq D\left(\right.$ Cust $\left._{x, y+1}\right) \leq \cdots \leq D\left(\right.$ Cust $\left._{x, n-1}\right)$. Where $D\left(\right.$ Cust $\left._{x, y}\right)$ is the distance between the Cust $x_{x}$ and Cust

For each customer, the ODV generates corresponding least distance customers in sorted order and rank the customers based on the distance, then it will moved to the Ordered Division Matrix (ODM) of order $n(n-$ 1) matrix $[1,7,8,13]$. 
Equi-begin (Eb):The beginning customer of the every individual is often same (i.e.) Cust 1 is fixed for all the individuals in the population. In our application, the beginning customer of the individuals is fixed therefore we have a tendency to are applying this technique $[1,8,13,14]$.

Variable diversity $(V d)$ :The Next customer in the individual is additional based mostly on the bax value, bax is an integer that selected within the range of best adjacent ( $b a$ ) value. The customer in the position of bax value is moved to following customer location of the individual $[1,8,13,14]$.

\section{Benchmark Dataset Instances For experimentation}

In this section a detailed study of the dataset used in the experimentation apart from the algorithm development and implementation. The bench mark instances for MDVRPTW obtained from VRPLIB and many more instances are available in the Library. The MDVRPTW instances that have been chosen for the experimentation are, pr01, pr02, pr03, pr07 and pr08[18].

These are the instance names we are going to use for our experimental analysis. Different authors provided different bench mark instances for MDVRPTW in neo research group. We are using Cordeau's benchmark instances; it contains 20 different instances of MDVRPTW[18].

\section{EXPERIMENTATION AND RESULT ANALYSIS}

All the implementations are done using MATLAB with MDVRPTW benchmark datasets obtained from VRPLIB (http://neo.lcc.uma.es/vrp/vrp-instances/ ). The MDVRPTW instances that have been chosen for the experimentation are pr01, pr02, pr03, pr07 and pr08. For each technique, the executions are carried out for 25 times and the average of each case has been considered for experimental analyses.

The analysis of ODV-EV Technique with different MDVRPTW instances is illustrates in Table $I$, this analysis is intended to explore the outcomes using ODVEV. From the table it is observed that the instance pr03 obtained higher result in case of best convergence rate and pr02 for worst convergence rate; and the best convergence rate of ODV-EV technique ranges from 96.61 to 94.99 for all the instances. Convergence diversity has obtained maximum of 33.67 for the instance pr07.

Table II shows the analysis of Gene Bank Technique with different MDVRPTW instances; the higher and lower values can be found in the Table II. The best and worst convergence rate of Gene bank technique has acquired maximum for a single instance pr02 and the overall convergence rate ranges from 95.50 to 89.31 . Instance pr08 has obtained best value 19.98 for Convergence diversity compared with all other instances.

\section{Best convergence rate}

The experimental analysis of the convergence rate is one of the significant performance analysis factors in GA.
Since Convergence Rate of an individual replicates quality of the individual w.r.t. the known optimal solution for the problem as shown in Fig.3, that the Best Convergence Rate of the EV technique than the Gene Bank population seeding technique.

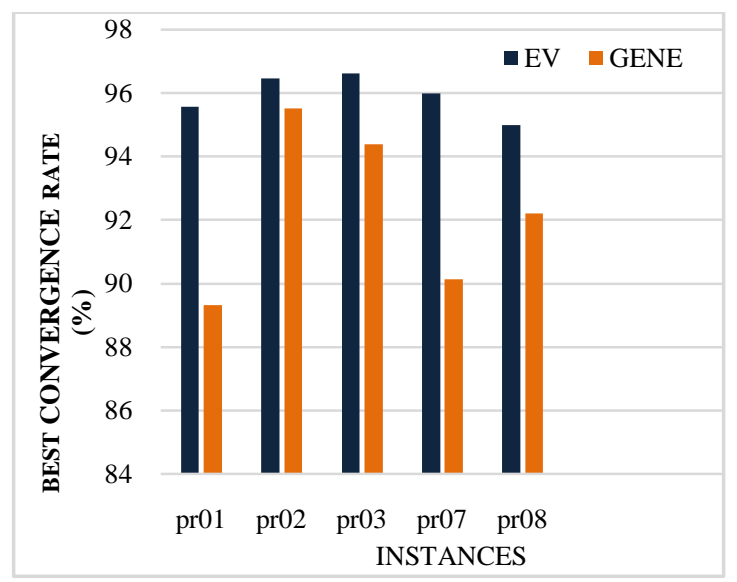

Fig. 3 Best Convergence Rate solution Generated by EV

The EV technique has showed extraordinary performance for all the five instances when compare to GB technique. The pr03 is exposed the higher convergence rate for ODV$\mathrm{EV}$ technique and pr08 has the lowest convergence rate in Gene Bank technique.

\section{Worst convergence rate}

Analyzing the Worst convergence rate, explores the quality of the individuals with least cost in the population. The worst convergence rate of the two population seeding techniques is plotted and showed in Fig. 4. The graph shows the Worst convergence rate Generated by EV technique.

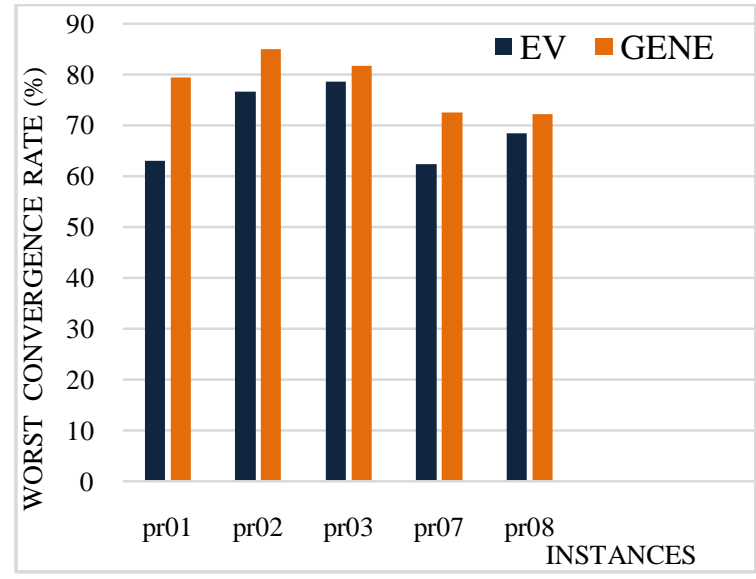

Fig. 4 Worst convergence Rate solution generated by EV

\section{Best Quality solution}

The Fig.5 shows the Best Quality Solution generated by EV Technique. The instance pr03 shows the Highest quality solution of 2489.95 and the lowest quality solution value of 1132.12 for the instance pr01. However the quality solution generated by GB is higher than EV for the instance pr03. 
International Journal of Advanced Research in Computer and Communication Engineering Vol. 3, Issue 11, November 2014

TABLE I

Result Analysis of MDVRPTW using ODV-EV Technique

\begin{tabular}{|c|c|c|c|c|c|c|c|c|c|}
\hline \multirow{2}{*}{ Sly } & \multirow{2}{*}{ Instance } & \multirow{2}{*}{$\begin{array}{c}\text { Optimal } \\
\text { Value }\end{array}$} & \multirow{2}{*}{ Capacity } & \multirow{2}{*}{ Route } & \multicolumn{2}{|c|}{ Quality Solution } & \multicolumn{2}{|c|}{$\begin{array}{c}\text { Convergence } \\
\text { Rate }(\%) \\
\end{array}$} & \multirow{2}{*}{ Diversity } \\
\hline & & & & & Best & Worst & Best & worst & \\
\hline 1 & pr01 & 1083.98 & 200 & 8 & 1132.12 & 1484.72 & 95.56 & 63.03 & 32.53 \\
\hline 2 & pr02 & 1763.07 & 195 & 12 & 1825.70 & 2176.66 & 96.45 & 76.54 & 19.91 \\
\hline 3 & pr03 & 2408.42 & 190 & 16 & 2489.95 & 2925.67 & 96.61 & 78.52 & 18.09 \\
\hline 4 & pr07 & 1423.35 & 200 & 12 & 1480.49 & 1959.76 & 95.99 & 62.31 & 33.67 \\
\hline 5 & pr08 & 2150.22 & 190 & 18 & 2257.94 & 2829.49 & 94.99 & 68.41 & 26.58 \\
\hline
\end{tabular}

TABLE II

Result Analysis of MDVRPTW using Gene Bank Technique

\begin{tabular}{|c|c|c|c|c|c|c|c|c|c|}
\hline \multirow{2}{*}{ Sly } & \multirow{2}{*}{ Instance } & \multirow{2}{*}{$\begin{array}{l}\text { Optimal } \\
\text { Value }\end{array}$} & \multirow{2}{*}{ Capacity } & \multirow{2}{*}{ Route } & \multicolumn{2}{|c|}{ Quality Solution } & \multicolumn{2}{|c|}{$\begin{array}{c}\text { Convergence } \\
\text { Rate }(\%)\end{array}$} & \multirow{2}{*}{ Diversity } \\
\hline & & & & & Best & Worst & Best & Worst & \\
\hline 1 & pr01 & 1083.98 & 200 & 8 & 1199.86 & 1308.07 & 89.31 & 79.32 & 9.98 \\
\hline 2 & pr02 & 1763.07 & 195 & 12 & 1841.35 & 2028.79 & 95.50 & 84.92 & 10.63 \\
\hline 3 & pr03 & 2408.42 & 190 & 16 & 2543.79 & 2848.96 & 94.37 & 81.70 & 12.67 \\
\hline 4 & pr07 & 1423.35 & 200 & 12 & 1563.80 & 1815.54 & 90.13 & 72.44 & 17.69 \\
\hline 5 & pr08 & 2150.22 & 190 & 18 & 2318.05 & 2747.62 & 92.19 & 72.21 & 19.98 \\
\hline
\end{tabular}

But during the experiment we found that the quality solution generated by EV was found to be best for most of the instances. The best quality solution of EV ranges from 2489.95 to 1132.12 as shown in the table I.

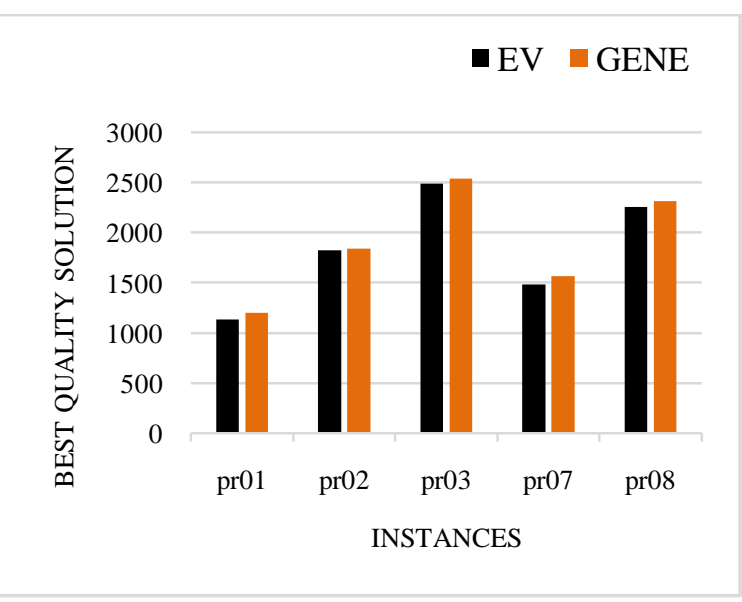

Fig. 5 Best Quality solution generated by EV

\section{Worst Quality solution}

The Fig.6 shows the Worst Quality Solution generated by EV Technique. The instance pr01 shows the lowest quality solution of 1308.07 in GB. However EV shows best for most of the instances during the experiment with more number of instances.

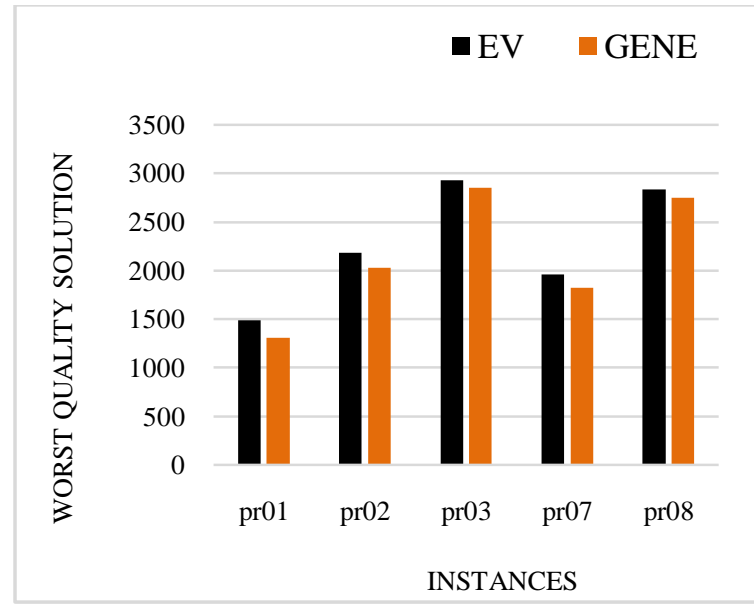

Fig. 6 Worst Quality solution generated by EV

\section{Convergence Diversity}

One of the reasons for observing the Convergence diversity of the individuals in the population is that to verify the variation between the best and worst individuals. Fig.7 shows Convergence diversity of the ODV-EV techniques reviled good result for the most of the instance and the Gene Bank technique exposed poor performance w.r.t the convergence diversity except for the instance pr08. 


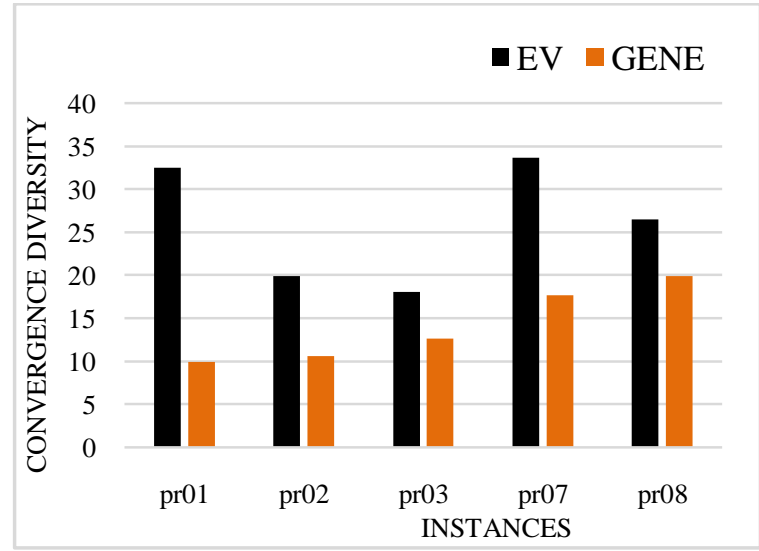

Fig. 7 Convergence Diversity solution generated by EV

\section{DISCUSSION}

In summary, the ODV-EV technique performs higher for many of the instances than the GB population seeding technique in terms of Convergence rate $(\%)$, Quality solution and Convergence Diversity. The quality solution for the best and worst case generated by EV technique shows best for the most of instances than the GB technique. The performance of GB is found to be good for few instances; however the EV gives better performance for most of the instances. The highest convergence rate generated by EV for the instance pr03 is 96.61 whereas GB gives 95.50 for the instance pr02. The proposed ODV based EV technique will give hundred percent best convergence solutions for large number of VRP instances. The convergence diversity of good and poor individuals among the population is generated and it's compared with proposed and existing GB technique in which EV perform better than the other. By using this proposed Technique we are going to develop an Enhanced Genetic Algorithm Model for extremely large number of instances and this process is under the review.

\section{CONCLUSION}

In this paper, the performance of Ordered Distance Vector (ODV) primarily based Equi-begin with Variable diversity (EV) population seeding technique is compared with Gene Bank (GB) population seeding technique. Experiments are performed using the Cordeau's benchmark instances with 20-customer instances and the benchmark datasets are obtained from the quality library VRPLIB were experimented using MATLAB tool. The experimental analysis primarily based on the performance issue of convergence rate, Convergence diversity and solution which shows higher performance for most of the VRP instances using ODV based EV techniques than the Gene Bank seeding technique. Population generated by this method is transferred for further process and forwarded to the enhanced genetic algorithm in the proposed technique and extended in our research.

\section{REFERENCES}

[1]. A. Ramalingam, K.Vivekanandan, and J. Sripriya, "Effective EV Population Seeding Technique for Vehicle Routing Problem with Time Windows using Genetic Algorithm", International Conference on Advances on Engineering and Technology (ICAET),October 2014,pp.19-22, ISBN:978-93-84209-60-5.

[2]. Behrouz Afshar-Nadjafi, Alireza Afshar-Nadjafi, "A constructive heuristic for time-dependent multi-depot vehicle routing problem with time-windows and heterogeneous fleet", Journal of King Saud University - Engineering Sciences (2014).

[3]. Surekha P, Dr.S.Sumathi, "Solution To Multi-Depot Vehicle Routing Problem Using Genetic",World Applied Programming, Vol (1), No (3), August 2011. 118-131 ISSN: 2222-2510.

[4]. Andrea Bettinelli, Alberto Ceselli, Giovanni Righini, "A branchand-cut-and-price algorithm for the multi-depot heterogeneous vehicle routing problem with time windows", Transportation Research Part C 19 (2011) 723-740.

[5]. G.Laporte, M.Gendreau, J.Potvin, and F.Semet, "Classical and modern heuristics for the vehicle routing problem", International Transactions in Operational Research, 7:285-300, 2000.

[6]. E. Falkenauer, "A hybrid grouping genetic algorithm for bin packing, Journal of Heuristics", 2:5-30, 1996.

[7]. P.VicterPaul,A.Ramalingam,R.Baskaran,P.Dhavachelvan, K.Vivekanandan, R.Subramanian and V.S.K.Venkatachalapathy, "Performance analysis on population seeding technique for genetic algorithms", P. Victer Paul et.al / International Journal of Engineering and Technology (IJET), July 2013. ISSN:0975-4024..

[8]. P. Victer Paul, A. Ramalingamb, R. Baskaran, P.Dhavachelvana,K.Vivekanandand,R.Subramanian, "A new population seeding technique for permutation-coded Genetic Algorithm: Service transfer approach", Journal of Computational Science 5 (2014) 277-297.

[9]. Vedat Toğan, Ayşe T. DaloğluAn improved genetic algorithm with initial population strategy and self adaptive member grouping,Computers \& StructuresVolume 86, Issues 11-12, June 2008, Pages 1204-1218

[10]. G. Nilay Yücenur, Nihan Çetin Demirel, “A new geometric shapebased genetic clustering algorithm for the multi-depot vehicle routing problem", Expert Systems with Applications 38 (2011) 11859-11865.

[11]. Suresh Nanda Kumar, Ramasamy Panneerselvam, "A Survey on the Vehicle Routing Problem and Its Variants", Intelligent Information Management, 2012, 4, pp.66-74.

[12]. Thomas Timmermann and Rene Schumann, "An approach to solve the multi depot vehicle routing problem with time windows (MDVRPTW) in static and dynamic scenarios", Sep 2008.

[13]. A.Ramalingam, K.Vivekanandan, "Solution Model For Capacitated Vehicle Routing Problem Using Genetic Algorithm", International Conference on CIIT-2014, August 2014, pp.178-190, ISBN:978981-09-1137-9, doi:10.3850/978-981-09-2579-6).

[14]. Claudio Contardoa, Rafael Martinelli, “A new exact algorithm for the multi-depot vehicle routing problem under capacity and route length constraints", Discrete Optimization 12 (2014) pp.129-146.

[15]. Syed Tauhid Zuhori, Zahrul Jannat Peya, Firoz Mahmud, “A Novel Three-Phase Approach for Solving Multi-Depot Vehicle Routing Problem with Stochastic Demand", Algorithms Research 2012, 1(4): 15-19 DOI: 10.5923 /j.algorithms. 20120104.01

[16]. Alireza Rahimi-Vahed, Teodor Gabriel Crainic, Michel Gendreau, Walter Rei, "Fleet-sizing for multi-depot and periodic vehicle routing problems using a modular heuristic algorithm", Computers \& Operations Research Sept 2012.

[17]. http://neo.lcc.uma.es/vrp/solution-methods

[18]. http://neo.lcc.uma.es/vrp/vrp-instances 